\title{
LA INVESTIGACIÓN SOBRE EL GÉNERO EN LA ESCUELA: NUEVAS PERSPECTIVAS TEÓRICAS
}

\section{Investigating gender in school: new theoretical approaches}

\section{La recherche du genre a l'école: des nouveaux perspectives théoriques}

María del Carmen Rodríguez MenÉndez y José Vicente PeÑa Calvo

Universidad de Oviedo. Facultad de Ciencias de la Educación. Departamento de

Ciencias de la Educación. C/. Aniceto Sela, s/n. 33005 Oviedo.

Correo-e: carmenrm@uniovi.es; vipe@uniovi.es

Fecha de recepción: enero de 2005

Fecha de aceptación definitiva: abril de 2005

BIBLID [(1130-3743) 17, 2005, 25-48]

RESUMEN

El artículo que se presenta realiza una exposición de la situación actual de la investigación educativa sobre el género. Se comienza con un análisis del modelo teórico que predominó durante las décadas de los años 70 y 80 en los países anglosajones, y que se prolongó en nuestro país hasta finales de los años 90 . Posteriormente, con objeto de superar las deficiencias mostradas por el modelo anterior, se explica la aparición y el desarrollo de un marco conceptual que ha amparado la mayor parte de la investigación educativa efectuada en los países de habla inglesa, desde principios de los 90 hasta nuestros días. Para ello, se presentan los supuestos teóricos que subyacen en el mismo y se comentan las diversas investigaciones educativas que se han efectuado.

Palabras clave: identidades de género, teoría de la socialización de los roles sexuales, feminismo postestructuralista, género y educación. 


\section{SUMMARY}

This paper develops an account of the current situation in education research about gender. It begins with an analysis of the theoretical approach which predominated over the 70s and 80s in Anglo-Saxon countries, and which extended its domain in Spain even over the 90s. In order to overcome the shortcomings showed by the former approach, a new developed conceptual framework it is explained. The latter has supported most of the education research in English-speaking countries from early 90 s until nowadays. In order to achieve this, the underlying theoretical assumptions are explained and several education researches are displayed.

Key words: gender identities, sex-role socialization theory, feminism poststructuralist, gender and education.

\section{SOMMAIRE}

L'article qui est présenté effectue un exposé de la situation actuelle de la recherche éducative sur le genre. On commence avec une analyse du modèle théorique qui a prédominé pendant les décennies des années de 70 et 80 dans les pays anglosaxons, et qui a été prolongé dans notre pays jusqu'à la fin des années 90. Postérieurement, dans le but de dépasser les insuffisances montrées par le modèle précedant, on explique l'apparition et le développement d'un cadre conceptuel qui a protégé le plus partie de la recherche éducative effectueé aux pays qui parlent anglais, dès le debut des 90 jusqu'à nos jours. À cet effect, on present les hypothèses théoriques qu'y sont sous-jacentes et on commente les diverses recherches éducatives qu'on a effectué.

Mots clef: identités de genre, théorie de la socialisation des rôles sexuels, féminisme poststructuraliste, genre et éducation.

\section{INTRODUCCIÓN}

En la década de los años 70, el feminismo comienza a interesarse por estudiar la construcción del género en el interior de las instituciones escolares; hasta ese momento se había prestado muy poca atención a esta variable en la investigación educativa (Hammersley, 2001, 27). Chicos y chicas eran educados por separado y, cuando ello no era así, la diferenciación por razón de género era usada formal e informalmente en las escuelas con propósitos curriculares y disciplinares. Esta distinción se asentaba en la idea de que hombres y mujeres presentaban claras diferencias biológicas, que los impelían a ejercer distintos roles en la vida social; en consecuencia las escuelas debían proporcionar un tratamiento curricular diferenciado para unos y otras con el objetivo de prepararlos para los roles que debían desempeñar. Sin embargo, a partir de la década de los años 70, y diez años más 
tarde en nuestro país, las actitudes comienzan a cambiar. Así, se emprenden algunas investigaciones educativas cuyo objetivo será analizar las diferentes experiencias escolares a las que niños y niñas son expuestos por razón de su género de pertenencia.

Tomando como base esta asunción, en las páginas que siguen, analizamos los diversos marcos conceptuales desde los que se han realizado las investigaciones sobre género y escuela. Comenzamos con el estudio de lo que se ha venido en llamar como "teoría de la socialización de los roles sexuales" (sex-role socialization theory). Al amparo de la misma, se han desarrollado la mayoría de las investigaciones en el área anglosajona durante las décadas de los años 70 y 80, así como casi todas las que se han efectuado en nuestro país desde mediados de los años 80 hasta finales de los 90. A continuación, analizamos las críticas realizadas a este marco teórico, en la medida en que este hecho supuso la aparición de un nuevo modelo que ha servido como base para la investigación educativa efectuada en los años 90 en los países anglosajones.

\section{GÉNERO Y ESCUELA: LA TEORÍA DE LA SOCIALIZACIÓN DE LOS ROLES SEXUALES}

Para Skelton (2001a, 43), dentro de la teoría de la socialización de los roles sexuales se deben incluir dos grandes modelos conceptuales surgidos al amparo de la psicología: la teoría del aprendizaje social y las aportaciones efectuadas desde la perspectiva cognitivista.

Así, las teorías del aprendizaje social señalan que los niños y las niñas aprenden modos apropiados de relacionarse con el mundo que les rodea a través de la observación, la imitación y su inclusión en procesos de interacción social basados en un sistema de recompensas y sanciones que refuerzan sus comportamientos. Se asume que la observación de conductas, tanto en modelos vivos como simbólicos (cine, televisión o libros), facilita el aprendizaje de la distinción entre los diversos patrones de conducta diferenciados por razón de género; de modo que niños y niñas aprenden por imitación los rasgos de conducta propios de cada género. En cuanto a la incidencia del reforzamiento, se señala que niños y niñas actúan de acuerdo con su género porque se les premia por hacer ciertas cosas y castiga por efectuar otras. De esta forma se concluye que realizan más a menudo las conductas recompensadas y adecuadas a su género y con una frecuencia menor aquellos comportamientos castigados, por ser inadecuados (Mischel, 1972). A partir de este proceso interactivo, las niñas aprenden e internalizan actitudes y conductas tales como el cuidado y la ayuda a los demás, la expresividad, la generosidad, etc.; mientras que los niños adquieren y demuestran características tales como la agresión, la independencia o la competitividad.

A partir de estos presupuestos teóricos, las teorías cognitivistas dan un paso más adelante, pues afirman que no sólo esos procesos están implicados en la adquisición de la identidad de género, sino que también es necesario tener en 
cuenta la organización cognitiva que el niño y la niña hacen de su mundo social. El punto de partida que se sugiere es que hay importantes componentes cognitivos implicados en la determinación de las actitudes relativas al papel de género. Así, por ejemplo, un concepto infantil que influye en la definición de actitudes sobre el rol de género es el conocimiento de su propia caracterización sexual o la generalización cognitiva de la misma hacia otros semejantes a él o ella. El niño y la niña aprenden un conjunto de reglas sobre lo que hacen los varones y lo que hacen las mujeres y se comportan de acuerdo con ellas. Por tanto, en el proceso de aprendizaje hay un componente de automotivación, pues unos y otras emprenden su propia socialización y seleccionan por su cuenta los comportamientos que deben aprender y ejecutar, sobre la base de las reglas correspondientes a las conductas apropiadas a su género. Abordan una valoración espontánea de sí mismos y de los demás; al tiempo que disponen de una tendencia cognitiva a atribuirse un valor a sí mismos, a comparar el suyo con los demás y a medir el de otras personas (Kolhberg, 1972). A partir de ello, se concluye que si bien los niños y las niñas pueden traspasar las fronteras de género pues son constructores activos de su propia identidad, también necesitan mantener una seguridad en su identidad de género, por lo que unos y otras refuerzan los patrones de género establecidos por el orden social. A partir de aquí, los desarrollos cognitivistas han formulado las distintas fases por las que transcurre el proceso de adquisición de la identidad de género, de modo semejante a como Piaget había determinado las distintas fases del desarrollo evolutivo (Fernández, 1988, 1996). Fases inamovibles, por las que todos y todas pasamos en los distintos momentos del ciclo vital.

Para C. Skelton (2001a) los modelos descritos se incluyen dentro de lo que algunas investigadoras e investigadores feministas han denominado como la "teoría de la socialización de los roles sexuales" (Connell, 1995, 2004; Davies, 1994, 2003; Francis, 2001; Francis y Skelton, 2001; Renold, 2001a, 2004; Jackson y Salisbury, 1996; A. Skelton, 1993; C. Skelton, 2001a, 2001b). La razón de este calificativo es que en ambos modelos subyacen una serie de planteamientos teóricos acerca de la construcción de la identidad de género, que serán la base para el desarrollo de una amplia variedad de investigaciones educativas. Sin lugar a dudas, la primera asunción que es común a ambos modelos es la idea de que el desarrollo de la identidad de género es un proceso de adquisición de los comportamientos correctos y adecuados para cada género, de modo que se produce una conformidad hacia las disposiciones básicas establecidas por el orden social. Sea por causa de los procesos de imitación y refuerzo u ocasionado por una valoración cognitiva de la propia persona, en ambos casos subyace la idea de que niños y niñas se muestran conformes con los papeles de género que les ha tocado desempeñar. Conformidad que tiene su base en la necesidad de presentarse ante el mundo como personas socialmente competentes.

Estas ideas llevan implícito el supuesto de que chicos y chicas adquieren los patrones de género de un modo no problemático, lo que genera una identidad unificada y coherente. A su vez, ello permite concluir que existen dos grupos diferenciados 
y homogéneos en sus características internas: el grupo de los "chicos" y el grupo de las "chicas", de modo que se produce un dominio de las categorías "niño", "niña", "hombre", "mujer". En consecuencia, se nos muestra una identidad de género fija, en donde los estereotipos de género son continuamente reproducidos por los agentes de socialización (familia, grupo de iguales, medios de comunicación, escuela). En este marco, y dado que la escuela se muestra como una institución socializadora clave en la niñez y adolescencia, comienzan a surgir una serie de estudios e investigaciones cuyo objetivo será analizar los medios y mecanismos utilizados en las mismas para reproducir los patrones de género existentes en el orden social.

Esto supuso que la investigación educativa se centrara en determinar las diferencias de género entre chicos y chicas en la institución escolar, concluyéndose que todos los chicos tenían las mismas oportunidades de acceso a todos los beneficios escolares y que todas las niñas estaban irremediablemente condenadas, por su condición femenina, a sufrir la desigualdad. Se asumía que la escuela, en cuanto ámbito de socialización secundaria, era una institución sumamente eficaz pues a través de ella se generalizaba la interiorización de las disposiciones básicas que socialmente se asignaban a cada género. La escuela, además, actuaba como agente de diferenciación genérica, contribuyendo a reforzar la situación desigual que niños y niñas tenían en la sociedad. Se señalaba que en ella habían desaparecido las diferencias formales en relación con el género, pero aún así seguían existiendo una multitud de mecanismos y procesos implícitos que reforzaban los estereotipos.

A partir de estos presupuestos, las diversas investigaciones emprendidas trataron de descubrir estos mecanismos. En este sentido, destacamos las investigaciones efectuadas con objeto de desvelar el sesgo masculino en los currícula oficiales y en los libros de texto. También fueron foco de análisis los procesos de interacción de niños y niñas en el aula y en el patio de recreo, lo que permitió concluir, entre otras cosas, que los niños acaparaban el espacio de juego, que había una profunda segregación por razón de género en las interacciones que se efectuaban y que chicos y chicas adoptaban, en las interacciones que establecían, actitudes y expectativas que se correspondían con el orden de género existente. Otro importante foco de la investigación se centró en analizar la dinámica de interacción profesorado/alumnado desde la perspectiva de género; concluyéndose que los patrones de interacción favorecían a los chicos, así como que los docentes efectuaban un tratamiento diferencial por razón de género que influía en la reproducción de los estereotipos ${ }^{1}$.

En suma, nos encontramos ante una profusa y compleja labor investigadora que permitió desvelar cómo los procesos de socialización escolar influían en la

1. En Rodríguez MENÉNDez (2003) se puede encontrar un resumen de las principales investigaciones efectuadas desde esta corriente en el ámbito español (acúdase al capítulo $5^{\circ}$ ). Asimismo, también se puede acudir a PeÑa Calvo y Rodríguez Menéndez (2002). 
configuración de pautas de comportamiento diferencial por razón de género, al tiempo que la escuela se percibió como una instancia socializadora que reforzaba los privilegios del género masculino.

\section{GÉNERO Y ESCUELA: NUEVAS PERSPECTIVAS TEÓRICAS}

Situándonos en el ámbito anglosajón debemos señalar que, a finales de la década de los 80 , comienzan a oírse algunas voces críticas en contra de los supuestos básicos que configuran la teoría de la socialización de los roles sexuales; así como de sus aplicaciones en el marco de las investigaciones educativas sobre el género $^{2}$. A partir de aquí los diversos autores y autoras que presentaremos en las páginas que siguen determinan que este modelo teórico simplifica enormemente el complejo desarrollo de la identidad de género, al asumir que dicha identidad es fija e inmóvil. Añaden que si se toma en consideración dicha teoría, los papeles de género se reproducen constantemente a través de los mecanismos de socialización anteriormente mencionados. Esta situación impediría reconocer algunos fenómenos importantes acaecidos en los últimos 20 años, tales como, por ejemplo, la creciente presencia femenina en el mercado laboral o el ascenso vertiginoso de las chicas en la pirámide educativa.

En suma, se concluye que la teoría de la socialización de los roles sexuales se muestra incapaz de explicar los cambios ocurridos en las relaciones de género, al asumir la existencia de unas identidades de género fijas, que son reproducidas constantemente a través de los diversos agentes e instituciones de socialización (Francis, 1999, 382; Renold, 2001a, 372). Se critica la asunción de que niños y niñas llegan a ser lo que la sociedad quiere que sean, disponiendo de poca o nula capacidad para pensar o sentir en modos distintos a los establecidos por el orden social. Un supuesto que comienza a ser cuestionado es el hecho de que niños y niñas adquieren la identidad de género a través de un proceso de observación y absorción acrítica de lo que les es ofrecido desde las instituciones sociales (MacNaugthon, 2000, 20).

2. Llegados a este punto, queremos dejar constancia de que a pesar de las críticas que se han realizado a la teoría de la socialización de los roles sexuales, de modo que en la actualidad se puede considerar que es un modelo superado, no debemos dejar de reconocer las importantes contribuciones que ha generado. Sin lugar a dudas, su aparición permitió acabar con las tesis esencialistas y biologicistas que afirmaban que las diferencias entre hombres y mujeres eran innatas y, por tanto, imposibles de modificar. Así, al sugerir que la construcción de la identidad de género es un proceso de carácter social, se establece por vez primera la posibilidad de que el statu quo en las relaciones entre los géneros pueda ser transformado. Por otra parte, tampoco nos es posible negar las aportaciones que han efectuado las diversas investigaciones educativas que se inscriben dentro de este modelo, de modo que han permitido poner en cuestión la idea de que la escuela es una institución neutral en la configuración de la identidad de género. 
A partir de esta crítica, surge un nuevo marco conceptual que supone la aplicación de los supuestos del feminismo postestructuralista al estudio de las relaciones entre género y escuela. En las páginas que siguen, determinaremos los supuestos teóricos que subyacen en el mismo para, a continuación, comentar las diversas investigaciones educativas que se han efectuado.

\subsection{Las investigaciones educativas efectuadas al amparo del postestructuralismo feminista}

La primera idea que debemos mencionar es la sugerencia de que la identidad de género es un fenómeno socialmente construido, permanentemente inacabado y sujeto a las múltiples y diversas influencias que ejercen los distintos marcos de acción dentro de los cuales las personas interaccionan en su vida cotidiana (Ali, 2003; Baxter, 2002; Cealey Harrison, 2001; Connell, 1995, 1998; Davies, 1994, 1997, 2003; Davies y Banks, 1992; Dillabough, 2001; Golden, 1996; Hallden, 1997; A. Jones, 1997; L. Jones, 1996; Jordan, 1995; Martino, 1995; Nilan, 2000; Pattman, Frosh y Phoenix, 1998; Reay, 2001; Renold, 1997, 2004; Salisbury y Jackson, 2003; Skelton, 2001a, 2001b; Swain, 2000; Volman y Ten Dam, 1998). Sin lugar a dudas esta asunción básica coincide con los supuestos básicos del postestructuralismo pues, como señala Hernández, en la década de los 90 la reflexión sobre la identidad y el sujeto ha introducido la duda en el concepto de esencia personal; la identidad se vuelve cada día más móvil, "formada y transformada continuamente en relación a las formas por las cuales somos representados e interpelados" (Hernández, 1999, 25).

En este sentido, Francis $(1999,383)$ reconoce que la teoría postestructuralista ha sido empleada por algunas investigadoras feministas como herramienta analítica en sus estudios. Concluye que la identidad de género no es fija, por lo que carece de sentido usar las categorías "femenino" y "masculino" en singular, pues ni hay una esencia femenina ni tampoco una esencia masculina. Se hace necesario reconocer la complejidad que subyace al proceso de "hacerse mujer" o "hacerse hombre", y las propias diferencias que hay entre las mujeres y entre los hombres; por lo cual las categorías "chico" y "chica", como las de "hombre" y "mujer", son problemáticas. La identidad de género es precaria, contradictoria y se encuentra en un proceso constante de reconstitución (A. Jones, 1997, 263), por lo que términos tales como variedad, heterogeneidad, ambigüedad o multiplicidad se hallan indisolublemente asociados al proceso de configuración del género (Mac An Ghaill, 1996; MacNaugthon, 2000; Parker, 1996; Renold, 2001a; C. Skelton, 1996). Se produce un movimiento "desde la identidad en tanto que nombre (y entonces estable y relativamente fija) a la identidad en tanto que verbo, siempre en proceso, tomando forma en y a través de las posibilidades discursivas por las que somos construidos" (Davies, 1997, 274).

En consecuencia, se asume que las personas somos sujetos activos en el proceso de construcción de nuestra identidad de género. De manera que "las niñas, y los 
niños, construyen y reconstruyen el mundo social a través de su (cambiante) comprensión del mismo, y a través de sus prácticas" (A. Jones, 1997, 262). A diferencia de lo establecido desde la teoría de la socialización de los roles sexuales, la identidad de género no es algo singularmente poseído o algo que «es" (estático), sino que es continuamente creado y recreado a partir de la interacción con los demás. De modo que se ha producido un cambio en la base de la comprensión del fenómeno: del estudio y análisis de los roles que hombres y mujeres aprenden de una forma más bien pasiva a través de la interacción social, se ha pasado a una visión de las identidades de género como relacionales, múltiples y diversas, estableciéndose los conceptos de "masculinidades" y "feminidades" (Otegui, 1999, 152; Renold, 2001a, 372-373; 2004, 249).

Asimismo, también se muestra que el género es una variable inextricablemente unida a otras, como la etnia o la clase social, provocando la emergencia de identidades variables y complejas (Ali, 2003; Archer y Yamashita, 2003; Casado, 1999; Connell, 1995; Francis y Skelton, 2001; Fraser, 1995; Griffiths, 1995; A. Jones, 1993; Lomas, 2004; Martino, 1995, 1999; Nayak, 2001; Nilan, 2000; Parker, 1996; Pattman, Frosh y Phoenix, 1998; Reay, 2001, 2002; C. Skelton, 1997, 2001a, 2001b, 2003; Swain, 2004). Desde esta perspectiva, se señala que las posiciones subjetivas disponibles para hombres y mujeres se modifican considerablemente si se tienen en cuenta características como la raza o la clase social. La atención a las múltiples y complejas influencias que unas variables ejercen sobre otras no hace sino subrayar la importancia que se concede a la "pluralidad de voces".

Igualmente, el predominio de la teoría postestructuralista también se deja sentir en la importancia concedida al discurso en tanto proceso a través del cual se configura la identidad de género. El discurso, entendido de forma amplia como modos de hablar, pensar o escribir, es importante porque estructura los caminos para comprender el mundo social. De modo que la identidad se resitúa y se negocia constantemente a través de las prácticas discursivas (Baxter, 2002; Davies, 1994; 1997, 2003; Davies y Banks, 1992; Francis, 1997a, 1997b, 1999, 2001; A. Jones, 1993, 1997; Reay, 2001; C. Skelton, 2001b); pero, al mismo tiempo, también se reconoce que hay determinados discursos que están más disponibles para los hombres que para las mujeres, y viceversa. En consecuencia, las prácticas discursivas definen las categorías y los dominios específicos que pueden ser dichos y hechos (Davies, 1994, 1997; Davies y Banks, 1992; Martino, 1995; Paechter, 2001), prescribiendo lo que es "normal" $\mathrm{O}$ "natural".

Tomando como punto de partida estas aseveraciones, también observamos otra particularidad común a todos los estudios. En concreto, nos referimos al tipo de metodología que se emplea en los mismos. Acabamos de subrayar la importancia que se concede al discurso en tanto instrumento prioritario a través del cual las personas nos presentamos ante el mundo social, entendemos dicho mundo, nos relacionamos con los demás y damos sentido a todo lo que nos rodea. En este marco, es fundamental dar voz a los distintos miembros de la comunidad educativa, en tanto que son los protagonistas de la investigación. En consecuencia, el 
estudio de casos basado en el uso de la observación participante y, fundamentalmente, de las entrevistas individuales y grupales fundamentará la línea metodológica a seguir. Lo que chicos y chicas dicen acerca de sus percepciones y experiencias tiene una importancia prioritaria, pues revela los modos a través de los cuales se produce la construcción social de las masculinidades y de las feminidades. En particular, señalamos el uso de grupos de discusión, mixtos o de un solo sexo, pues esta técnica se revela especialmente eficaz al permitir la creación de un espacio en el que las personas pueden hablar libremente acerca de los que es importante y significativo para ellas. Se aprecia que sólo actuando de este modo es posible comprender cómo chicas y chicos se construyen y se posicionan a sí mismos dentro del complejo entramado de relaciones que se establecen en las instituciones educativas.

A partir de estos elementos comunes, observamos una profusa labor investigadora que se ha efectuado, primordialmente, en los países anglosajones, en particular Gran Bretaña, Australia y Nueva Zelanda. Dado que el volumen de lo escrito y publicado es muy amplio, hemos decidido establecer una tipología de los estudios, tomando como variable de referencia la mayor o menor proximidad conceptual con los supuestos básicos que se acaban de establecer. Sin lugar a dudas, la elaboración de una tipología tiene sus peligros pues hay autores y autoras que resultan difíciles de categorizar, al tiempo que en otros casos los supuestos teóricos que sustentan sus estudios se han ido matizando con el paso del tiempo. Siendo conscientes de estas limitaciones nos hemos decidido por su realización, dada su claridad didáctica. Así, hemos denominado a las categorías del siguiente modo: postestructuralismo puro, postestructuralismo moderado y postestructuralismo reconstituido.

\subsubsection{La fragmentación de la identidad: el postestructuralismo puro}

La razón que nos ha impulsado a usar este calificativo es que las investigaciones incluidas en esta categoría han tenido por objeto analizar las complejas interacciones que se establecen entre el género y otras variables tales como la raza o la clase social. En consecuencia, y a diferencia de los modelos siguientes, se establece la imposibilidad de fijar y determinar una serie de categorías de género. Se indica que la construcción de la identidad es altamente compleja y se plantea como cuestión clave la diferencia, de modo que las experiencias de los grupos étnicos o de las distintas clases sociales influyen de modo determinante en la configuración de la identidad y en las experiencias educativas que tienen chicos y chicas. Las masculinidades y las feminidades son apreciadas como plurales, en la medida que siempre se expresan a través de la etnia y de la posición de clase. Desde su punto de vista, una plena comprensión de las relaciones que se establecen entre género y educación solamente es posible si el género es tratado como un proceso plural que encuentra expresión en relación a otros caminos a través de los cuales chicos y chicas son posicionados (Phoenix, 2001, 127). 
A partir de ello han surgido una variedad de estudios que han tenido por objeto analizar las diversas experiencias educativas de ser una chica o un chico blanco, asiático, afroamericano, afrocaribeño, de clase trabajadora, de clase media, etc. La complejidad de los cruzamientos posibles ha generado que la mayoría de los estudios se centren en analizar las prácticas escolares de un subgrupo particular en un contexto también determinado ${ }^{3}$. En este sentido, situamos básicamente dos tipos de análisis: por un lado, señalamos los exámenes de carácter más general, que tratan de observar las intersecciones entre la etnia y el género (Connolly, 1998; Mac An Ghaill, 1996; Nayak, 2001; Phoenix, 2001), o entre el género y la clase social (Lucey, 2001; Nayak, 2003; Skeggs, 1997). Asimismo, en otras ocasiones, nos encontramos con estudios más específicos que determinan relaciones más complejas entre las distintas variables; sería el caso, por ejemplo, de algunas monografías que han explorado la construcción de la masculinidad, en el contexto escolar, de chicos provenientes de familias de clase trabajadora, con el objetivo de estudiar las diferencias por razón de etnia (Archer, Pratt y Phillips, 2001; Archer y Yamashita, 2003); también se han observado los esfuerzos por analizar a las chicas que pertenecen a la clase trabajadora y que además tienen problemas para ser definidas como pertenecientes a una etnia determinada (Ali, 2003).

En los estudios apreciamos el énfasis que se sitúa en la complejidad del proceso de constitución de la identidad de género. Se percibe que la finalidad es observar, a partir de lo que las personas implicadas relatan, los caminos, a veces tortuosos, a través de los cuales la etnicidad, la clasę social y el género configuran la propia identidad; y todo ello analizado a la luz del marco escolar. Sin lugar a dudas, éstas son las investigaciones educativas que más acentúan la idea de que la producción de la identidad de género es una actividad mucho más compleja de lo que se creía desde la teoría de la socialización de los roles sexuales, enfatizándose la idea de que el proceso de configuración del self es un proyecto constantemente incompleto. Por esta razón, son los estudios que más se acercan a la idea de fluidez y fragmentación propia del discurso feminista postestructuralista.

\subsubsection{La existencia del dualismo y la jerarquía de género: el postestructuralismo moderado}

Sin lugar a dudas, la mayor parte de las investigaciones educativas efectuadas se inscriben dentro de esta corriente. En todas ellas se reconoce la fluidez y el cambio en el proceso de configuración de la identidad de género. No obstante, también se percibe la necesidad de tener en cuenta los constreñimientos sociales que

3. En el texto sólo se citarán aquellas publicaciones que han sido leídas por los autores del artículo. El número de estudios y trabajos efectuados es mucho más amplio. Sin embargo, una primera aproximación a esta temática la proporcionan los trabajos citados. Acúdase a ellos como primera referencia; sin embargo, si lo que se quiere es completar y profundizar en estas cuestiones es interesante la lectura de las referencias bibliográficas que acompañan a dichos estudios. 
determinan los modelos de deseabilidad social para hombres y mujeres. En este sentido, debemos destacar, en primer lugar, los trabajos de Bronwyn Davies, pues fue una de las primeras investigadoras que aplicó los supuestos del postestructuralismo feminista a la interpretación de las relaciones entre género y educación. Por esta razón, la influencia que ha ejercido sobre los escritos editados a lo largo de la década de los años 90 es patente y notoria. Si bien Davies ha evolucionado, con el paso de los años, hacia la necesidad de subrayar la fragmentación del proceso de construcción de la identidad, en sus publicaciones siempre subyace el deseo de explicar la contradicción y tensión que se origina entre la necesidad de resistirse y, al mismo tiempo, acomodarse a los patrones de género existentes en el orden social.

En su primer libro importante desde la perspectiva postestructuralista, Davies (1994, original publicado en 1989) comienza efectuando una crítica enérgica de la teoría de la socialización de los roles sexuales, al señalar que ésta se fundamenta en la asunción de que niños y niñas construyen una identidad de género unificada y coherente, a través de unas relaciones no problemáticas con el mundo social que les rodea. A continuación, establece los fundamentos de una teoría de gran potencia explicativa, para lo cual toma como punto de referencia el análisis de los procesos de configuración de la identidad de género en niños y niñas que asisten a educación infantil, y usando como metodología de referencia el estudio de caso. Como resultado de ello concluye que el desarrollo de la identidad de género es un proceso en el cual niños y niñas construyen discursos a través de las lecturas e interpretaciones que efectúan de las experiencias que viven. No obstante, añade que unos y otras no son totalmente libres para construir determinados discursos y, por ende, algunas identidades alternativas; pues Davies asume que algunos discursos son más poderosos que otros porque están más disponibles, son más deseables o más placenteros para los niños y las niñas.

A diferencia de la teoría de la socialización de los roles sexuales, esta autora no asume que los niños y niñas estén aprisionados en las categorías "hombre" y "mujer" respectivamente; sino que, por el contrario, señala que en el aprendizaje de lo que es ser un miembro coherente de la sociedad unos y otras toman de forma activa su género asignado, a través de caminos que no son necesariamente compatibles con los modos socialmente aceptables (Davies, 2003, original publicado en 1993). Como señala Davies, hay diversos caminos para llegar a ser hombres y mujeres (enfatizo el plural), y en un mundo ideal cada persona debería tener acceso a esos diversos caminos de configuración. No obstante, también reconoce que en el mundo real esto no es así, de modo que la oposición binaria hombre/mujer ejerce su influencia en el proceso de configuración de la identidad. Subraya que la sociedad ha elaborado un discurso de género que divide a hombres y mujeres en dos categorías opuestas (dualismo hombre/mujer) y que niños y niñas de corta edad se identifican a sí mismos como pertenecientes al género correcto. De modo que Davies señala que unos y otras realizan un trabajo de mantenimiento de la categoría 
"género" ${ }^{4}$. Sin embargo, y éste es el matiz esencial en su trabajo, señala que ese trabajo de mantenimiento es terriblemente complejo y contradictorio. Reconoce que ante la presión para el mantenimiento de las categorías, niños y niñas pueden hacer dos cosas: o acomodarse o resistir. Señala que en la mayoría de las ocasiones, dado que no se dispone de una identidad fija y coherente, oscilan entre ambos polos, añadiendo a la ecuación la necesaria contradicción en el proceso identitario. Indica que la resistencia y el mantenimiento configuran un proceso dual que es central en el estudio de la vida social de los niños y niñas, pues a través de él, el género es constantemente creado y mantenido (Davies, 2003, 23).

Si bien se señala la existencia del dualismo, la autora no deja de reconocer la contradicción en el proceso de modo que, como señala MacNaugthon (2000, 2223) de una forma muy acertada, las grandes aportaciones de Davies han sido la necesidad de dar respuesta a una serie de interrogantes que no habían sido tomados en consideración hasta el momento, a saber: ¿cuáles son los medios a través de los cuales niños y niñas se resisten/acomodan a los discursos dominantes sobre el género?, ¿cómo reaccionan unos y otras cuando se les presentan discursos contradictorios?, ¿cómo se comportan cuando eligen entre discursos dominantes o alternativos? y, por último, ¿qué aspectos del orden social están influyendo para que elijan o rechacen un discurso dominante/alternativo? El trabajo que realiza en el contexto de tres escuelas infantiles trata de dar respuesta a esos interrogantes (para un análisis más detenido acúdase a Davies, 1994)

Otro hito importante en el desarrollo de las investigaciones educativas postestructuralistas lo supuso la aparición de los trabajos de Connell (1989, 1995, 1998). Dicho autor instituye la noción de "masculinidad hegemónica". Dicha expresión, así como el marco teórico que la fundamenta, será asumido como base conceptual de muchas de las investigaciones educativas que se efectuarán desde el

4. Este trabajo de mantenimiento de la categoría "género" lo observa, con especial empeño, cuando se les leían los denominados "cuentos feministas". Éstos se caracterizan por presentar historias tradicionales a través de personajes que se comportan de modos alternativos. En este caso, con frecuencia, niños y niñas critican el comportamiento de los protagonistas, lo que demuestra la fuerza de las categorías de género.

5. Subrayando el carácter de la resistencia pero también el de mantenimiento de las categorías de género señalamos un estudio efectuado por WESTLAND (1993). Esta autora analiza los discursos de una serie de niños y niñas con edades comprendidas entre los 9 y los 11 años, organizados en una serie de grupos de discusión, y tomando como temática de discusión los roles de género que aparecen en los cuentos tradicionales. Si bien la autora reconoce la fragilidad en la construcción de la identidad, pues las respuestas que niños y niñas dan a los roles de género establecidos por los cuentos tradicionales son variadas, diversas y contradictorias, también concluye que una alta proporción de las niñas de esta edad son "lectoras resistentes" $(1993,242)$, estando dotadas de la capacidad de criticar y manipular a su antojo las imágenes de género que aparecen en los cuentos tradicionales. En oposición a ello, la autora reconoce que muchos de los chicos entrevistados se adaptan más al patrón estereotipado, pues tienen menos incentivos para traspasar las fronteras de género. Para la autora, la posición de superioridad que ostentan en el sistema de relaciones de género provoca que no vean necesario modificar una situación que les privilegia. 
feminismo postestructuralista. Comienza Connell $(1989,1995)$ afirmando que la construcción de la masculinidad es un proceso colectivo, de modo que varias masculinidades son construidas en dependencia unas de otras, y también en relación a las feminidades. A partir de esto, establece que dichas identidades no son estáticas, sino que están histórica y espacialmente situadas, al tiempo que una forma hegemónica de masculinidad ("masculinidad hegemónica") tiende a erigirse como dominante, ejerciendo gran influencia y autoridad en un contexto y tiempo determinados (Connell, 1995, 1998; C. Skelton, 2001a, 2001b; Swain, 2000). En el mismo sentido, podemos hablar de la existencia de una afeminidad hegemónica.. Naturalmente, la feminidad y la masculinidad hegemónica son, en muchos sentidos, frágiles e inseguras, y necesitan ser mantenidas y defendidas constantemente, de modo que la contradicción y la tensión son notas que caracterizan sus procesos de configuración (Hickey y Fitzclarence, 1999; Kenway y Fitzclarence, 1997; Nilan, 2000; Pattman, Frosh y Phoenix, 1998; Salisbury y Jackson, 2003; Swain, 2000, 2002, 2003). A pesar de ello, se sostienen en prácticas discursivas dominantes, que influyen en la definición de la identidad personal de los chicos y las chicas. En oposición a ellas se encuentran las "otras masculinidades" y las "otras feminidades", de modo que aquellos que se "desvían" del ideal hegemónico pueden incurrir en costes emocionales y sociales, siendo calificados de "marginados" O "subordinados" (Connell, 1995; Pattman, Frosh y Phoenix, 1998; Renold, 2004; Salisbury y Jackson, 2003; C. Skelton, 2001a, 2001b).

La aparición de este marco teórico tendrá una importancia prioritaria, en la medida que su concepto de "masculinidad hegemónica" abrirá la puerta al estudio de los complejos procesos escolares en los que se configuran las distintas masculinidades (y feminidades). Señala Connell (1998) que cada colegio dispone de su propio régimen de género, el cual está formado por expectativas, reglas, rutinas y un orden jerárquico. Todo ello crea diferentes repertorios de acción y tiene profundos efectos en las condiciones a través de las cuales chicos y chicas configuran su identidad personal. Asimismo, se señala que en la cúspide del régimen de género de la escuela se encuentra la masculinidad hegemónica, la cual se construye en oposición a la feminidad y a otras formas alternativas de vivenciar el ser masculino.

A partir de aquí han surgido multitud de investigaciones que han tenido por objeto analizar la construcción de las distintas masculinidades en el interior de las escuelas, hasta el punto que esta temática puede ser considerada como el ámbito de estudio más prolífico desde mediados de la década de los 90 hasta nuestro días. Si bien la exploración de la configuración de la masculinidad en el espacio de la escuela ya había sido realizada con anterioridad en algunas investigaciones de carácter etnográfico, caso del estudio efectuado por P. Willis (1977), o el más reciente realizado por Askew y Ross (1988. Trad. cast. 1991). A partir de la década de los 90 y como consecuencia del impacto de los trabajos de Connell $(1989,1995)$, se redefine el tipo de discurso elaborado y, en consecuencia, se abrirán nuevos horizontes en el tratamiento de los estudios sobre la construcción de la masculinidad en la escuela. 
Dado que no es objeto del artículo hacer un análisis exhaustivo de todas las investigaciones, presentamos los supuestos comunes que las fundamentan. En primer lugar, todos los estudios son coincidentes al señalar que no todas las masculinidades triunfan y que éstas se interrelacionan a partir de una compleja trama de relaciones e intercambios sociales que tiene su expresión en el contexto escolar (Martino, 1999; Nilan, 2000; Swain, 2000, 2002). De modo que las diferencias entre unos chicos y otros se sitúan a distintos niveles, haciendo referencia, por ejemplo, al modo en que hablan, la manera de vestirse, los juegos y deportes que se prefieren, la música que se escucha o el modo de presentar el cuerpo ante los demás. En suma, los recursos sociales (habilidades interpersonales), culturales (moda, música...), físicos (deporte), intelectuales (logro académico) y económicos (dinero disponible) que cada uno de los chicos es capaz de acumular y presentar ante los demás determinarán su estatus y ayudarán a establecer su identidad masculina (Swain, 2004). En relación a este punto, las investigaciones también son proclives a señalar la importancia del grupo de iguales, pues la configuración de la identidad masculina es una "empresa colectiva" (Swain, 2004, 171) y está unida a la adquisición de estatus dentro del grupo de compañeros. Éste provee un espacio donde se ponen en juego prácticas sociales y discursivas que sirven para validar y amplificar la masculinidad hegemónica (Archer y Yamashita, 2003; Connell, 1989; Ivinson y Murphy, 2003; Kenway y Fitzclarence, 1997; Pattman, Frosh y Phoenix, 1998; Redman, 1996; C. Skelton, 1997; Swain, 2002). De hecho se afirma que una de las dimensiones más importante para los chicos es ganar popularidad y estatus dentro del contexto escolar, por lo que su búsqueda está firmemente unida al logro de una forma aceptable de masculinidad (Swain, 2003, 302).

Todo ello permitirá que cada chico se posicione en un lugar dentro de la jerarquía de género existente en la escuela, de modo que quien no logre configurarse desde posiciones cercanas a la masculinidad hegemónica será calificado de "gay" o "marica". De modo que "parecer" o "ser" ligeramente distinto a la norma puede colocar a un chico en un estatus inferior (Epstein, 1997; Jackson, 2003; Kehily, 2001; Kehily y Nayak, 1997; Martino, 1999; Parker, 1996; Pattman, Frosh y Phoenix, 1998; Redman, 1996; Renold, 2000, 2001a, 2003, 2004; C. Skelton, 2000, 2001b; Swain, 2000, 2002, 2003).

Los estudios también subrayan que la masculinidad hegemónica se fundamenta en la ostentación de una conducta heterosexual. En este sentido, la mayoría de las investigaciones, sea de forma marginal o preponderante, han mostrado cómo las prácticas que sostienen la masculinidad hegemónica están inequívocamente unidas a las nociones dominantes sobre la heterosexualidad. Desde esta perspectiva, la sexualidad se percibe como una forma de control y resistencia, al tiempo que de refuerzo de particulares versiones de la masculinidad (Epstein, 1997; Nayak y Kehily, 1997; Redman, 1996; Renold, 2000, 2003; Swain, 2003). Así, se concluye que para ser un "verdadero" chico se ha de ser heterosexual; hasta el punto que la heterosexualidad se percibe como una norma a seguir dentro de la escuela. De modo que los significados y prácticas que a ella se asocian son valoradas como 
estrategias usadas por los adolescentes para negociar sus relaciones en el seno de la escuela; al tiempo que les permiten adoptar una identidad de género estable y segura a los ojos de los demás. Si bien, como señalan diversos autores (Kehily y Nayak, 1997, 152; Renold, 2000, 319; 2003, 182), el proceso de "llegar a ser" heterosexual es mucho más complejo y contradictorio de lo que a simple vista pudiera parecer.

En oposición a esta profusión investigadora, que ha tenido por objeto analizar los complejos y frágiles procesos a través de los cuales los chicos se hacen hombres, no ha habido un interés semejante por considerar lo que le ocurre a las chicas. Desde nuestro punto de vista, esta situación se ha originado por la coincidencia temporal en el surgimiento de las investigaciones educativas postestructuralistas y el creciente interés por el estudio de los procesos sociales implicados en la construcción de la masculinidad en el contexto escolar. Situación que ha provocado una merma en el interés por estudiar la configuración de la feminidad en la escuela. No obstante, debemos destacar los esfuerzos realizados por Emma Renold (2000, 2001b), la cual analiza la construcción de la sexualidad femenina en el contexto de la escuela primaria. Señala la existencia de diversas feminidades, aquellas que se adecuan a los estereotipos dominantes sobre la sexualidad de las chicas (feminidad hegemónica), y aquellas que construyen su identidad sexual de modos alternativos, transformando las ideas dominantes sobre el cuerpo femenino y su sexualidad. Si bien Renold (2000) reconoce las dificultades para resistirse a las categorías de género, pues la mayoría de las chicas se adecuan al estereotipo, también es cierto que abre la puerta a la posibilidad de que la configuración de la feminidad sea un proceso complejo y contradictorio. En un sentido muy similar, Renold (2001b) también trata de desmontar una de las asunciones básicas que se ha asumido en el estudio de las relaciones entre género y educación. En particular, la idea de que el alto rendimiento académico de las chicas es una cuestión no problemática, a diferencia de lo que ocurre con los chicos. En oposición a ello, señala que las niñas que manifiestan altos logros académicos ocupan una posición no femenina dentro del aula, puesto que se oponen a la feminidad hegemónica. A partir de ello, analiza las tensiones, contradicciones y complejidades entre la necesidad de adecuarse al estereotipo y el deseo de comportarse en modos que trascienden las fronteras de género. Manteniendo una línea muy similar, señalamos el estudio de Liz Jones (1996), quien analiza los diversos discursos que sobre la feminidad manifiestan niñas de 4 años, revelando la conciliación que se produce entre los discursos dominantes y alternativos sobre la feminidad.

En conclusión, podemos decir que los estudios que se inscriben dentro de lo que hemos denominado como postestructuralismo moderado subrayan la necesidad de reconocer la contradicción que subyace a los procesos de configuración de la identidad de género, una identidad siempre fluida y en constante proceso de negociación. Pese a ello también se muestran de acuerdo con la idea de que no todas las masculinidades y las feminidades gozan del mismo valor en el contexto social: La existencia de las categorías de género (dualismos, en palabras de Davies) 
permite el establecimiento de una jerarquía o régimen de género en el que las distintas feminidades y masculinidades se muestran en relación las unas con las otras. Los modelos de deseabilidad social que se presentan a los niños reconocen la prioridad manifiesta de la masculinidad y la feminidad hegemónica. Esta situación genera un "trabajo de mantenimiento de las categorías" (en palabras de Davies) que se caracteriza por la confluencia continua de la resistencia y de la acomodación, en un proceso constante de negociación que genera una identidad frágil, y hasta cierto punto inconsistente.

Se parte de la idea de un sujeto "ni determinado, ni libre, sino ambas cosas de forma simultánea" (A. Jones, 1997, 263. La traducción es nuestra). Así, por una parte, el discurso se percibe como un instrumento necesario para trascender las categorías de género, pues se establece la posibilidad de que las personas generemos discursos transgresores que nos ayuden a resistir y modificar los papeles socialmente asignados. Es decir, se reconoce la idea de que somos sujetos activos que podemos modificar la fuerza del discurso dominante. Sin embargo, por otro lado, también se señala la fuerza que ejerce dicho discurso en la configuración de la propia identidad. Se subraya que las personas usamos diversas estrategias para acomodarnos, pero al mismo tiempo resistir, a los discursos de género establecidos por el orden social. De modo que se intenta ofrecer una explicación que permita incorporar las nociones de resistencia y contradicción, que tan problemáticas han sido para la teoría de la socialización de los roles sexuales, al tiempo que se reconoce la fuerza de determinados discursos dominantes en el proceso de configuración de la identidad de género.

\subsubsection{Coherencia e identidad: el postestructuralismo reconstituido}

Sin lugar a dudas, será Becky Francis la investigadora que defina los parámetros de este modelo. Tomando en cuenta los postulados generales del postestructuralismo, Francis realiza una crítica de algunos de los supuestos que lo configuran. A partir de ello define un nuevo modelo caracterizado por la asunción básica de que los discursos de género dominantes que son establecidos por el orden social tienen un peso mayor en el proceso de configuración de la identidad de género que los discursos de resistencia.

El aspecto más destacado de su teoría es que aún incidiendo en la idea de complejidad propia de la corriente postestructuralista, también cuestiona "la noción de que no hay coherencia de la identidad todo el tiempo" (Francis, en London Feminist Salon Collective, 2004, 28). De este modo, Francis mantiene que algunos elementos de nuestra identidad pueden ser estables a través de las diferentes interacciones con el entorno. Ello implica la necesidad de buscar algún tipo de consistencia de la identidad, al tiempo que retenemos las nociones de cambio y fluidez propias de la corriente postestructuralista. Nótese que la autora habla de la idea de consistencia de la identidad, para afirmar que la coherencia es posible dado que 
las personas, en nuestra vida diaria, realizamos las distintas actividades pensando y sintiendo que somos sujetos únicos que tenemos nuestra propia personalidad (Francis, 2001, 69). Es más, la autora critica el presupuesto postestructuralista que reconoce que las personas nos sentimos sujetos coherentes, en tanto que este sentimiento es una ilusión causada por el discurso individualista propio del pensamiento modernista occidental. Para Francis, la consistencia de la identidad no es una quimera ilusoria, sino algo lleno de sentido y significación.

Se mantiene firme en la postura de creer que si bien es necesario reconocer los múltiples y complejos caminos a través de los cuales nos convertimos en "hombres" o "mujeres", también es necesario señalar que la plena aceptación de la existencia de una identidad fragmentada e incoherente puede romper la narrativa feminista (Francis, 1999, 388). En relación a este punto, también señala que las posiciones enmarcadas dentro de la teoría postestructuralista muestran grandes dificultades para elaborar marcos de referencia globales que den cuenta de la pervivencia de los dualismos de género. Si todo es múltiple, diverso y contradictorio los esfuerzos por definir marcos teóricos que expliquen el mantenimiento del orden social de género pueden ser estériles. Es cierto que muchas de las teorías surgidas al amparo del postestructuralismo han permitido avanzar en el conocimiento del proceso de construcción del género, pues se ha señalado la necesidad de acentuar la complejidad del propio proceso. Así, las diferencias entre las categorías "hombre" y "mujer" (nótese el singular) son menores de las que previamente se habian supuesto; no obstante esto no significa asumir que dichas diferencias no existan. En consecuencia, señala que las ideas feministas que han sido universales, tales como por ejemplo la situación de opresión de la categoría "mujer" con respecto de la categoría "hombre", entran en contradicción con un discurso que, como el postestructuralista, quiere deconstruir las grandes narrativas o relatos de la modernidad (Francis, 1999, 385). El postestructuralismo subraya que no hay una "experiencia de vida femenina" universal, dada la multiplicidad que acompaña al proceso identitario; lo que para Francis resulta muy peligroso pues no permite establecer marcos de referencia globales que den cuenta, por ejemplo, de las relaciones de poder entre "mujeres" y "hombres". Es más, llega a establecer que estos supuestos postestructuralistas al privar a las teorías feministas de una serie de herramientas conceptuales que expliquen la subordinación por razón de género, contribuyen a su mantenimiento y reproducción (Francis, 1999, 387, 390; 2001, 65, 68).

A partir de aquí sus investigaciones educativas han sido un intento de reconciliar los supuestos postestructuralistas con la noción de coherencia del self. En este sentido, usa el concepto de "dualismos de género" o "construcción dicotómica" para mostrar las formas predominantes de masculinidad y feminidad que se manifiestan en las instituciones escolares. Coincide de este modo con Davies o Connell, autores determinantes del postestructuralismo moderado, pero la diferencia estriba en que Francis da un mayor peso al discurso dominante de mantenimiento de las categorías de género, al tiempo que reconoce la coherencia de la identidad. A partir de estas asunciones básicas, Francis concluye que la categoría masculina se 
define como silly-selfish (tonto-egoísta), la cual se asocia a atributos tales como la inmadurez, el desorden o la travesura; mientras que la categoría femenina se define como sensible-selflessness (sensata-desinteresada) (Francis, 1997b, 1998). A partir de aquí, Francis analiza la comprensión que tienen niños y niñas de los dualismos de género, concluyendo que en la mayoría de los casos unos y otras se adjetivan a sí mismos y al género opuesto en base a las categorías mencionadas. Si bien, la autora reconoce que las fronteras de género se pueden cruzar y expone ejemplos de cómo ello es posible (Francis, 1997b, 189; 1998, 36-38), también reconoce las grandes dificultades que se presentan para hacerlo, enfatizando la pervivencia del discurso dominante. Además, subraya que el mantenimiento del dualismo facilita que los niños mantengan las posiciones de poder en el interior de la clase (Francis, 1997b, 187-188), al tiempo que también reconoce que en grupos mixtos es donde este poder se manifiesta de modo más acuciante y donde el mantenimiento de las categorías se hace más poderoso (Francis, 1997b, 183, 190).

En suma, la postura de Francis supone la asunción teórica de que la identidad de género se constituye y reconstituye a través de las prácticas discursivas; pero, al mismo tiempo, también reconoce que las personas preferimos determinados discursos, que están marcados por el régimen de género existente en la sociedad. Como señala C. Skelton (2001b, 175), Francis nos muestra una teoría que intenta articular las tensiones entre el reconocimiento de las múltiples posiciones que las personas podemos adoptar y el reconocimiento de que somos sujetos con una identidad coherente, no fragmentada ${ }^{6}$.

\section{CONCLUSIÓN}

En las páginas precedentes hemos efectuado un recorrido por los diversos modelos teóricos que han estudiado las relaciones entre género y educación.

6. Debemos dejar constancia de dos autoras que, desde nuestro punto de vista, se encuentran muy próximas a los postulados de Francis. En primer lugar, señalamos a Alison Jones (1993), aunque ella se inscribe dentro de la corriente postestructuralista, un análisis detenido de su trabajo nos permite apreciar la importancia que concede a la necesidad de combinar unidad y diversidad en la explicación de la configuración de la identidad de género. Asimismo, también reconoce que el discurso de la resistencia resulta muy complejo para los niños y niñas, de modo que aunque las fronteras de género se pueden traspasar la complejidad de este proceso inhibe las posibilidades de éxito. También subrayamos los trabajos de Diane REAY (2001), pues aunque manifiesta una posición más ambigua al reconocer que las relaciones de género que se establecen en el marco de la institución escolar se encuentran en proceso constante de renegociación y recomposición (lo que la haría próxima al postestructuralismo moderado, cuestión que en un trabajo publicado en 2002 se aprecia con más claridad); señala además que, a pesar de ello, el régimen de género se mantiene, pues la oposición y la jerarquía son notas que caracterizan las relaciones entre las niñas y los niños. Del mismo modo, en su estudio acerca de la construcción de las feminidades en el interior de las escuelas de primaria (REAY, 2001), concluye que el discurso de continuidad pesa más que el de transgresión. 
Hemos expuesto cómo estas distintas corrientes están conectadas con diferentes supuestos teóricos acerca del proceso de construcción de la identidad de género.

En consecuencia, hemos analizado la teoría que ejerció un peso dominante en las décadas de los años 70 y 80 en los países anglosajones, y en nuestro país hasta finales de la década de los años 90. La teoría de la socialización de los roles sexuales, con su conceptualización de la identidad de género en tanto que constructo fijo y no problemático, así como su estudio del proceso de construcción de dicha identidad en tanto que mecanismo de asunción acrítica de los papeles de género, definió los análisis educativos efectuados durante un largo periodo de tiempo.

La aparición del paradigma postestructuralista marcó un punto de inflexión en las reflexiones sobre la identidad de género, así como en las investigaciones que se emprendieron en el ámbito educativo. La variedad de estudios efectuados desde esta perspectiva teórica en el ámbito anglosajón, nos ha llevado a intentar sistematizarlos a partir de la construcción de una tipología. Por ende, se han reconocido tres modelos distintos que, tomando en común el reconocimiento de los supuestos básicos del feminismo postestructuralista, suponen distintas aproximaciones al estudio de la identidad de género y, en consecuencia, al análisis de las escuelas en tanto agentes socializadores clave en ese proceso de configuración. Hemos descrito un postestructuralismo puro, que asume la fragmentación del proceso de configuración de la identidad de género como un aspecto central en sus análisis. Esta asunción ha generado una multitud de estudios que han tenido por objeto interpretar las complejas relaciones que el género mantiene con otras variables tales como la etnia o la clase social, y todo ello explicado a la luz de las interacciones escolares.

A continuación, hemos analizado los estudios inscritos en el postestructuralismo moderado, reconociendo que éste ha sido el modelo en el que se pueden inscribir la mayor parte de las investigaciones educativas efectuadas. Dicho modelo reconoce la contradicción que subyace a los procesos de configuración de la identidad de género, una identidad siempre fluida y en constante proceso de negociación. Pese a ello también se muestran de acuerdo en la idea de que no todas las masculinidades y las feminidades gozan del mismo valor en el contexto social, lo que permite reconocer la existencia de las categorías de género.

Por último, señalamos la posición más controvertida de Francis; un posicionamiento que ha generado la puesta en cuestión de la idea mantenida desde el feminismo postestructuralista de que la identidad de género está fragmentada. Su postura, con la que los firmantes de este artículo están plenamente de acuerdo, no supone una vuelta a la visión de la identidad de género como categoría fija. Asume que construimos el género de manera distinta dependiendo de las interacciones en las que estamos inmersos; no obstante, también reconoce que al presentarnos ante los demás mantenemos cierta coherencia del self (Francis, 2001, 70-71). Coherencia que ella demuestra en la necesidad de reconocer el mantenimiento de los dualismos de género y la importancia que los mismos tienen en la vida de las personas. 


\section{BIBLIOGRAFÍA}

ALI, S. (2003) "To be a girl»: culture and class in schools, Gender and Education, 15 (3), 269283.

ARcher, L.; PratT, S. D. y Phillips, D. (2001) Working-class men's constructions of masculinity and negotiations of (non) participation in higher education, Gender and Education, 13 (4), 431-449.

ARCHER, L. y YAMASHITA, H. (2003) Theorising Inner-city masculinities: "race", class, gender and education, Gender and Education, 15 (2), 115-132.

Askew, S. y Ross, C. (1991) Los chicos no lloran. El sexismo en educación. Barcelona, Paidós. (Trabajo original publicado en 1988).

BAXTER, J. (2002) A juggling act: a feminist post-structuralist analysis of girls' and boys' talk in the secondary classroom, Gender and Education, 14 (1), 5-19.

Casado Aparicio, E. (1999) A vueltas con el sujeto del feminismo, Política y Sociedad, 30, 73-91.

Cealey Harrison, W. (2001) Truth is slippery stuff, en Francis, B. y Skelton, C. (eds.). Investigating gender. Contemporary perspectives in education. Buckingham, Open University Press, 52-64.

ConNel, R. W. (1989) Cool guys, swots and wimps: the interplay of masculinity and education, Oxford Review of Education, 15 (3), 291-303.

- (1995) Masculinities: knowledge, power and social change. Cambridge, Polity Press. (Traducción al castellano: Masculinidades. México, UNAM, 2003).

- (1998) Enseñar a los chicos: nuevas investigaciones sobre la masculinidad y estrategias de género para la escuela, Kikiriki, 47, 51-68.

- (2004) Gender. Cambridge, Polity Press. (Trabajo original publicado en 2002).

CONNOLLY, P. (1998) Racism, gender identities and young children. London, Routledge.

DAVIES, B. (1994) Sapos y culebras y cuentos feministas. Los niños de preescolar y el género. Madrid, Cátedra. (Trabajo original publicado en 1989).

- (1997) The subject of post-structuralism: a reply to Alison Jones, Gender and Education, 9 (3), 271-283.

- (2003) Shards of glass. Children reading \& writing beyond gendered identities. Revised edition. Creeskill, Hampton Press. (Trabajo original publicado en 1993).

DAvies, B. y BANKS, Ch. (1992) The gender trap: a feminist poststructuralist analysis of primary school children's talk about gender, Journal of Curriculum Studies, 24 (1), 1-25.

Dillabough, J.-A. (2001) Gender theory and research in education: modernist traditions and emerging contemporary themes, en Francis, B. y SkELTON, C. (eds.). Investigating gender. Contemporary perspectives in education. Buckingham, Open University Press, 11-26.

EPSTEIN, D. (1997) Boyz' own stories: masculinities and sexualities in schools, Gender and Education, 9 (1), 105-115.

FERNÁNDEZ, J. (coord.) (1988) Nuevas perspectivas en el desarrollo del sexo y del género. Madrid, Pirámide.

- (coord.) (1996) Varones y mujeres. Desarrollo de la doble realidad del sexo y del género. Madrid, Pirámide.

FrancIS, B. (1997a) Discussing discrimination: children's construction of sexism between pupils in primary school, British Journal of Sociology of Education, 18 (4), 519-532.

- (1997b) Power plays: children's constructions of gender and power in role plays, Gender and Education, 9 (2), 179-191. 
- (1998) Oppositional positions: children's construction of gender in talk and role plays based on adult occupation, Educational Research, 40 (1), 31-43.

- (1999) Modernist reductionism or post-structuralist relativism: can we move on? An evaluation of the arguments in relation to feminist educational research, Gender and Education, 11 (4), 381-393.

- (2001) Beyond postmodernism: feminist agency in educational research, en Francis, B. y Skelton, C. (eds.). Investigating gender. Contemporary perspectives in education. Buckingham, Open University Press, 65-76.

Francis, B. y SKELTON, C. (eds.) (2001) Investigating gender. Contemporary perspectives in education. Buckingham, Open University Press.

Fraser, N. (1995) Multiculturalidad y equidad entre los géneros, Revista de Occidente, 173, 35-55.

GOLDEN, J. (1996) Critical imaginations: serious play with narrative and gender, Gender and Education, 8 (3), 323-335.

GRIFFITHS, M. (1995) Making a difference: feminism, post-modernism and the methodology educational research, British Educational Research Journal, 21 (2), 219-235.

HALLDEN, G. (1997) Competence and connection: gender and generations in boys' narratives, Gender and Education, 9 (3), 307-316.

HAMMERSLEY, M. (2001) Obvious, all too obvious? Methodological issues in using sex/gender as a variable in educational research, en Francis, B. y SKELTON, C. (eds.). Investigating gender. Contemporary perspectives in education. Buckingham, Open University Press, 27-38.

HERNÁNDEZ, F. (1999) Consideraciones sobre el sujeto y la identidad en la educación escolar, Kikiriki, 51, 21-26.

Hickey, C. y FitzClarenCE, L. (1999) Educating boys in sport and physical education: using narrative methods to develop pedagogies of responsibility, Gender and Education, 4 (1), 51-62.

IVINSON, G. y MuRPHY, P. (2003) Boys don't write the romance: the construction of knowledge and social gender identities in English classrooms, Pedagogy, Culture and Society, 11 (1), 89-111.

JACKSON, C. (2003) Motives for "laddishness" at school: fear of failure and fear of the feminine, British Educational Research Journal, 29 (4), 583-598.

JACKSON, D. y SALISBURY, J. (1996) Why should secondary schools take working with boys seriously, Gender and Education, 8 (1), 103-116.

JONES, A. (1993) Becoming a "girl": post-structuralist suggestions for educational research, Gender and Education, 5 (2), 157-166.

- (1997) Teaching post-structuralist feminist theory in education: student resistances, Gender and Education, 9 (3), 261-269.

JONES, L. (1996) Young girls' notions of feminity, Gender and Education, 8 (3), 311-321.

JORDAN, E. (1995) Fighting boys and fantasy play: the construction of masculinity in the early years of school, Gender and Education, 7 (1), 69-86.

KeHILY, M. J. (2001) Issues of gender and sexuality in schools, en FrancIs, B. y SKelton, C. (eds.). Investigating gender. Contemporary perspectives in education. Buckingham, Open University Press, 116-125.

KEHILY, M. J. y NAYAK, A. (1997) Lads and laughter': humour and the production of heterosexual hierarchies, Gender and Education, 9 (1), 69-87. 
KENWAY, J. y FITZCLARENCE, L. (1997) Masculinity, violence and schooling: challenging poisonous pedagogies, Gender and Education, 9 (1), 117-133.

KOLHBERG, L. (1972) Análisis de los conceptos y actitudes infantiles relativos al papel sexual desde el punto de vista del desarrollo cognitivo, en MaccoBy, E. E. Desarrollo de las diferencias sexuales. Madrid, Marova, 61-147. (Trabajo original publicado en 1966).

LOMAs, C. (2004) Los chicos también lloran. Identidades masculinas, igualdad entre los sexos $y$ coeducación. Barcelona, Paidós.

LONDON Feminist Salon Collective (2004) The problematization of agency in postmodern theory: as feminist educational researchers, where do we go from here?, Gender and Education, 16 (1), 25-33.

LuCEY, H. (2001) Social class, gender and schooling, en Francis, B. y SKELTON, C. (eds.). Investigating gender. Contemporary perspectives in education. Buckingham, Open University Press, 177-188.

MAC AN GHallL, M. (1996) What about the boys?: schooling, class and crisis masculinity, Sociological Review, 44 (3), 381-397.

MacNaughton, G. (2000) Rethinking gender in early childbood education. Sydney, Allen \& Unwin.

MARTINO, W. (1995) Deconstructing masculinity in the English classroom: a site reconstituting gendered subjectivity, Gender and Education, 7 (2), 205-220.

- (1999) "Cool boys", "party animals", "squids" and "poofters": interrogating the dynamics and politics of adolescent masculinities in school, British Journal of Sociology of Education, 20 (2), 239-263.

Mischel, W. (1972) Las diferencias sexuales en las conductas desde el punto de vista del aprendizaje social, en MACCOBY, E. E. Desarrollo de las diferencias sexuales. Madrid, Marova, 37-60. (Trabajo original publicado en 1966).

NAYAK, A. (2001) "Ice white and ordinary": new perspectives on ethnicity, gender and youth cultural identities, en FRANCIS, B. y SkelTON, C. (eds.). Investigating gender. Contemporary perspectives in education. Buckingham, Open University Press, 139-151.

- (2003) "Boyz to men": masculinities, schooling and labour transitions in de-industrial times, Educational Review, 55 (2), 147-159.

NiLAN, P. (2000) You're hopeless I swear to God: shifting masculinities in classroom talk, Gender and Education, 12 (1), 53-68.

Otegui, R. (1999) La construcción social de las masculinidades, Política y Sociedad, 32, 151160.

PAECHTER, C. (2001) Using poststructuralist ideas in gender theory and research, en Francis, B. y SKELTON, C. (eds.). Investigating gender. Contemporary perspectives in education. Buckingham, Open University Press, 41-51.

PARKER, A. (1996) The construction of masculinity within boys' physical education, Gender and Education, 8 (2), 141-157.

Pattman, R.; Frosh, S. y Phoenix, A. (1998) Lads, machos and others: developing "boy centred' research", Journal of Youth Studies, 1 (2), 125-142.

Peña Calvo, J. V. y Rodríguez Menéndez, M. ․ㅡ C. (2002) Identidades esquemáticas de género en la escuela: a propósito del primer aniversario de la muerte de Pierre Bourdieu, Teoría de la Educación. Revista Interuniversitaria, 14, 235-263.

PhoenIx, A. (2001) Racialization and gendering in the (re)production of educational inequalities, en Francis, B. y SKelTON, C. (eds.). Investigating gender. Contemporary perspectives in education. Buckingham, Open University Press, 126-138. 
REAY, D. (2001) The paradox of contemporary feminities in education: combining fluidity with fixity, en FranCIS, B. y SKELTON, C. (eds.). Investigating gender. Contemporary perspectives in education. Buckingham, Open University Press, 152-163.

- (2002) Shaun's story: troubling discourses of white working-class masculinities, Gender and Education, 14 (3), 221-234.

REDMAN, P. (1996) Curtis love Ranjit: heterosexual masculinities, schooling and pupils' sexual cultures, Educational Review, 48 (2), 175-182.

RENOLD, E. (1997) "All they've got on their brains is football". Sport, masculinity and the gendered practices of playground relations, Sport, Education and Society, 2, 5-23.

- (2000) Coming out: gender, (hetero)sexuality and the primary school, Gender and Education, 12 (3), 309-326.

- (2001a) Learning the "hard" way: boys, hegemonic masculinity and the negotiation of learner identities in the primary school, British Journal of Sociology of Education, 22 (3), 369-385.

- (2001b) Square-girls, feminity and the negotiation of academic success in the primary school, British Educational Research Journal, 27 (5), 577-588.

- (2003) If you don't kiss me, you're dumped: boys, boyfriends and heterosexualised masculinities in the primary school, Educational Review, 55 (2), 179-194.

- (2004) Other boys: negotiating non-hegemonic masculinities in the primary school, Gender and Education, 16 (2), 247-266.

RODRÍGUEZ MENÉNDEZ, M. C. (2003) La configuración del género en los procesos de socialización. Oviedo, KRK.

SAlISBURY, J. y JACKSON, D. (2003) Challenging macho values. Practical ways of working with adolescent boys. London/New York, Routledge Falmer/Taylor \& Francis group. (Trabajo original publicado en 1996).

SkEGGS, B. (1997) Formations of class and gender. London, Sage.

SKELTON, A. (1993) On becoming a male physical education teacher: the informal culture of students and the construction of hegemonic masculinity, Gender and Education, 5 (3), 289-303.

SKELTON, C. (1996) Learning to be "tough": the fostering of maleness in one primary school, Gender and Education, 8 (2), 185-197.

- (1997) Primary boys and hegemonic masculinities, British Journal of Sociology of Education, 18 (3), 349-369.

- (2000) A passion for football: Dominant masculinities and primary schooling, Sport, Education and Society, 5 (1), 5-18.

- (2001a) Schooling the boys. Masculinities and primary education. Buckingham, Open University Press.

- (2001b) Typical boys? Theorizing masculinity in educational settings, en Francis, B. y SkELTON, C. (eds.). Investigating gender. Contemporary perspectives in education. Buckingham, Open University Press, 164-176.

- (2003) Male primary teachers and perceptions of masculinity, Educational Review, 55 (2), 195-209.

SwaIN, J. (2000) "The money's good, the fame's good, the girls are good": The role of playground football in the construction of young boys' masculinity in a junior school, British Journal of Sociology of Education, 21 (1), 95-109.

- (2002) The right stuff: fashioning an identity clothing in a junior school, Gender and Education, 14 (1), 53-69. 
- (2003) How young schoolboys become somebody: the role of the body in the construction of masculinity, British Journal of Sociology of Education, 24 (3), 299-314.

- (2004) The resources and strategies that 10-11-year old boys use to construct masculinities in the school setting, British Educational Research Journal, 30 (1), 169-185.

VOLMAN, M. y TeN DAM, G. (1998) Equal but different: contradictions in the development of gender identity in the 1990s, British Journal of Sociology of Education, 19 (4), 529-545.

WESTLAND, E. (1993) Cinderella in the classroom. Children's responses to gender roles in fairy-tales, Gender and Education, 5 (3), 237-249.

WILLIS, P. (1977) Aprendiendo a trabajar: o cómo los chicos de clase obrera eligen trabajos de clase obrera. Madrid, Akal. 